\title{
Üreter tașı için üreterorenoskopik cerrahi yapılan erkek ve kadın hastalarda JJ stent yerleștirilmesinin seksüel fonksiyonlar üzerine etkisi
}

\author{
The effect of JJ stent placement on sexual functions in male and female \\ patients who underwent ureterorenoscopic surgery for ureteral stones
}

Arif Kalkanlı®, Cem Tuğrul Gezmiș®

\section{öz}

\begin{abstract}
AMAÇ: Çalışmada amacımız kadın ve erkek hastalarda üreterorenoskopik üreter taşı tedavisi (URS) sonrası JJ stent tatbikinin seksüel fonksiyonlar üzerinde etkisini araştırmaktır.

GEREÇ ve YÖNTEMLER: Mayıs 2020 - Temmuz 2021 tarihleri arasında URS yapılan 48 kadın ve 76 erkek hasta prospektif çalışmaya dahil edildi. İlk değerlendirmede hastalara kreatinin, tam idrar tetkiki, idrar kültürü ve üriner CT yapıldı. Hastaların yaş, BMI ve taş yükleri kaydedildi. Erkek hastalar için Uluslararası Erektil İşlev Formu-5 (IIEF-5), kadın hastalar için Kadın Cinsel İşlev Ölçeği (FSFI) formları işlem öncesi, işlem sonrası birinci ve üçüncü ay olmak üzere üç kez dolduruldu. JJ stent takılan hastaların ikinci ölçümleri stent çekilmesi için başvuru anında yapıldı. İşlem sonrası birinci ayda tüm hastalara tekrar CT çekildi ve rezidü taş kalıp kalmadığı tespit edildi.
\end{abstract}

BULGULAR: Erkek hastaların IIEF-5 skorları ortalaması işlem öncesi $24,45 \pm 8$ iken birinci ayda $18,47 \pm 6,73$ 'e düşmüş üçüncü ayda ise $23,95 \pm 8,55$ 'e çıkmışıı $(\mathrm{p}<0,01)$. Kadın hastaların ortalama FSFI değerleri ise işlem öncesi $17,2 \pm 7,7$ iken birinci ayda $12 \pm 5,12$ 'ye düşmüş, üçüncü ayda ise $17,8 \pm 7,9$ 'a yükselmiştir $(\mathrm{p}<0,01)$. Her iki cinsiyette JJ stent takılan hastaların takılmayanlara göre sorgulama formu skorlamaları ilk ölçüme göre birinci ay ölçümlerinde istatistiksel anlamlı şekilde daha fazla düşmüştür (IIEF-5: \%23,33 vs \%31,12; FSFI: \%21,25 vs $\% 30,88$ ).

SONUÇ: URS yapılan hastalarda geçici seksüel fonksiyon gelişebileceği bilinmelidir. JJ stent takılan hastalarda seksüel fonksiyonlarda kötüleşme daha fazla meydana gelmektedir.

Anahtar Kelimeler: Erkek seksüel disfonksiyonu, kadın seksüel disfonksiyonu, üreterorenoskopi, JJ stent

\section{ABSTRACT}

OBJECTIVE: The purpose of this study is to investigate the effects of JJ stent placement following ureterorenoscopic ureter stone (URS) treatment on sexual functions of male and female patients.

MATERIAL and METHODS: 48 female and 76 male patients who underwent URS surgery between May 2020 and July 2021 were included in the study. During the first examination, creatinine level, urianalysis results, and urinary CT findings were recorded for each patient as well as their age, BMI and stone burden. International Index of Erectile Fuction (IIEF-5) and Female Sexual Function Inventory (FSFI) were answered for a total number of three times (first preoperatively, and then on the postoperative first and third month) by male and female patients, respectively. Second measures of patients for whom JJ stent was used were taken when they applied for the removal of the stent. All patients received CT on the postoperative first month to assess the presence of possible residue stones.

RESULTS: Mean IIEF-5 scores of male patients was $24.45 \pm 8$ preoperatively, decreasing to $18.47 \pm 6.73$ on the first postoperative month, and increasing to $23.95 \pm 8.55$ on the third postoperative month $(\mathrm{p}<0.01)$. For female patients, mean FSFI value was $17.2 \pm 7.7$ preoperatively, decreasing to $12 \pm 5.12$ on the first month, and increasing to $7.8 \pm 7.9$ on the third $(\mathrm{p}<0.01)$. Inquiry form scores of both male and female patients to whom JJ stent was placed showed a significantly higher decrease compared to patients for whom JJ stents were not used (IIEF-5:23.33\% vs 31.12\%; FSFI: $21.25 \%$ vs $30.88 \%$ ).

CONCLUSION: Temporary sexual dysfunction is likely to develop following URS procedures. Our results indicate that placement of JJ stent increases the severity of the sexual dysfunction.

Keywords: Male sexual dysfunction, female sexual dysfunction, ureterorenoscopy, JJ stent
Taksim Eğitim ve Araştırma Hastanesi, Üroloji Kliniği, İstanbul, Türkiye

Yazışma Adresi/ Correspondence:

Dr. Arif Kalkanlı

Teah Üroloji Kliniği Sıraselviler Cad Beyoğlu İstanbul, Türkiye

Tel: $\quad$ +095067402254

E-mail: arifkalkanli@gmail.com

Geliš/ Received: $\quad 22.08 .2021$

Kabul/ Accepted: $\quad 25.09 .2021$

\section{Giriș}

Prevalansı \%20'ye varan ve en sık görülme yaşı aynı zamanda bireylerin seksüel olarak en aktif oldukları 20-50 yaş grubu olan üriner sistem taş hastalı̆̆ı, üroloji pratiğinde en sık karşılaşılan hastalıklardan biridir. ${ }^{[1-3]}$ Taş hastalıklarının önemli bir kısmını üreter taşları oluşturmaktadır. Üreter taşları için tedavi stratejisi oluşturulurken taşın boyutu, 
yerleşimi, sayısı, hastanın anatomik özellikleri ve cerrahın deneyimi göz önünde bulundurulur. ${ }^{[3]}$ Üreterorenoskopi (URS), son 4 dekattır üreter taşlarının tedavisinde ekstrakorporal şok dalga ile taş kırma (SWL), açık cerrahi ve laparoskopik cerrahi ile birlikte en sık kullanılan tedavi modalitesidir. ${ }^{[4,5]}$ URS yüksek başarı oranları ve düşük morbidite oranlarıyla üroloji pratiğinde yerleşik minimal invaziv bir tedavi yöntemidir. ${ }^{[6]} \mathrm{Bu}$ nedenle semi-rijit URS ile küçük distal üreter taşlarından, fleksibl URS ile büyük renal pelvis taşlarının tedavisine kadar endikasyonları genişlemiştir. ${ }^{[6,7]}$

Hem erkek hem de kadın seksüel fonksiyonlarının sağlıklı olabilmesi için bireylerin vasküler, nöronal, endokrin ve genitorüriner sistemleri uyum içinde çalışmalıdır. ${ }^{[8,9]}$ Bununla birlikte psikososyal faktörlerin de bireyin yaşam kalitesinde önemli bir rol oynamakta olan cinsellik üzerine etkileri vardır. ${ }^{[10,11]}$ Şimdiye kadar erkek ve kadınlarda diabetes mellitus, nörolojik hastalıklar, depresyon, anksiyete, radyoterapi gibi birçok risk faktörünün seksüel fonksiyonları olumsuz etkilediği saptanmıştır. [12-17] Üroloji pratiğinde uygulanan radikal prostatektomi ve sistektomi gibi onkolojik operasyonların seksüel hayat üzerine olumsuz etkileri uzun süredir bilinirken, son zamanlarda prostat biyopsisi, SWL gibi işlemlerin de cinsel fonksiyonlar üzerinde olumsuz etkilerinin olabileceği bildirilmiştir. ${ }^{[18,19]}$

Üreteral stentler obstrüksiyon durumunda böbrekten mesaneye kadar idrar akışını sağlarlar ve böylece böbreğin korunmasına yardımcı olurken aynı zamanda idrar ekstravazasyonunu önlerler. ${ }^{[20]}$ URS sonrası rutin olarak JJ kateter tatbik edilmesi önerilmese de üreteral travma, rezidüel taş olması, kanama, perforasyon, üriner enfeksiyon ve gebelik gibi durumlarda JJ stent tatbik edilmektedir. ${ }^{[3]}$

URS sonrası JJ kateter tatbik edilen hastalarda postoperatif dönemde ağrı, hematüri, dizüri ve diğer üriner sisteme ait semptomların daha fazla görüldüğü bildirilmiştir. ${ }^{[20-23]}$ Bu gibi semptomlar hastalarda ajitasyon, anksiyete ve hatta uykusuzluk gibi psikolojik faktörler yaratabilmektedir. ${ }^{[20-23]} \mathrm{Bu}$ prospektif gözlemsel klinik çalışmamızdaki amacımız URS yapilan hastalarda JJ kateter tatbikinin URS sonrası oluşan geçici seksüel disfonksiyon üzerine etkisini araştırmaktır.

\section{GEREÇ ve YÖNTEMLER}

\section{Hasta Seçimi}

Mayıs 2020 - Temmuz 2021 tarihleri arasında üreter taşı için üreteroskopi yapılan 18-50 yaş aralığında 188 hasta çalışmaya dahil edildi. Gaziosmanpaşa Eğitim ve Araştırma
Hastanesi Klinik Araştırmalar Etik Kurulu'ndan onay (166/2020) alındıktan sonra tüm hastalara çalışma hakkında bilgi verildi ve onam formları alındı. Hastalar ile yapılan görüşme sonrası seksüel disfonksiyon sebebiyle tedavi alan (farmokolojik, psikoterapi, şok dalga tedavisi vb.), seksüel aktif olmayan (son 12 aydır cinsel ilişki öyküsü olmayan), üriner sistem cerrahi öyküsü, malignite öyküsü, nörojen mesane, aşırı aktif mesane, böbrek taşı, mesane taşı, üretra darlığı, kronik prostatit ve benign prostatik obstrüksiyonu olan hastalar çalışmadan çıkarıldı. Düzenli kontrole gelmeyen, rezidü taşı kalan, taşı kırılamayan ve ek girişim gerektiren hastalar da çıkarıldıktan sonra 48 kadın ve 76 erkek hasta ile çalışma tamamlandı. Tüm hastalara ilk başvuruda tam idrar tetkiki, kreatinin, idrar kültürü, üriner CT gerçekleştirildi. Hastaların yaş, BMI ve taş yükü (taş yükü, taşın en uzun horizontal çapının, taşın en uzun dikey çapıyla çarpılmasıyla belirlenen iki boyutlu alan olarak tanımlandı) kaydedildi (Şekil 1).

\section{Üreterorenoskopi Tekniği}

Medikal tedavi ile spontan pasajın gerçekleşmediği semptomatik üreter taşı hastaları çalışmaya dahil edildi. İdrar kültürü ile idrarın steril olduğu gösterildi. Ameliyat sabahı düz üriner sistem grafisi çekildikten sonra tüm hastalara sistoskopi yapıldı. Kılavuz telin üretere skopik görüş altında yerleştirilmesinin ardından, lazer veya pnömatik litotriptör kullanılarak taş parçalanması için 7,5 Fr rijit üreteroskop yerleştirildi. İşlem sonrası ekstravazasyonu dışlamak için retrograd pyelogram çekildi. JJ stentlerin yerleştirilmesi uluslararası kılavuzların önerdiği şekilde, komplikasyon riski yüksek olan hastalarda (üreter travması, rezidüel parçalar, kanama, perforasyon veya intraoperatif enfekte görünüm) ve tüm şüpheli durumlarda uygulandı. JJ stent takılan hastaların stentleri 3-6 hafta sonra sistoskopi eşliğinde çekildi.

\section{Seksüel Fonksiyonların Değerlendirilmesi Ve Takip}

Erkek hastalar için Uluslararası Erektil İşlev Formu-5 (IIEF-5), kadın hastalar için Kadın Cinsel İşlev Ölçeği (FSFI) formları işlem öncesi, işlem sonrası birinci ve üçüncü ay olmak üzere üç kez dolduruldu. ${ }^{[24,25]} \mathrm{JJ}$ stent takılan hastaların ikinci ölçümleri stent çekilmesi için başvuru anında yapıldı. İşlem sonrası birinci ayda tüm hastalara tekrar CT çekildi ve rezidü taş kalıp kalmadığı tespit edildi.

\section{İstatiksel Analiz}

Veriler, Windows SPSS sürüm 22 (SPSS, Inc., Chicago, IL, ABD) kullanılarak analiz edildi. Demografik veriler ve 


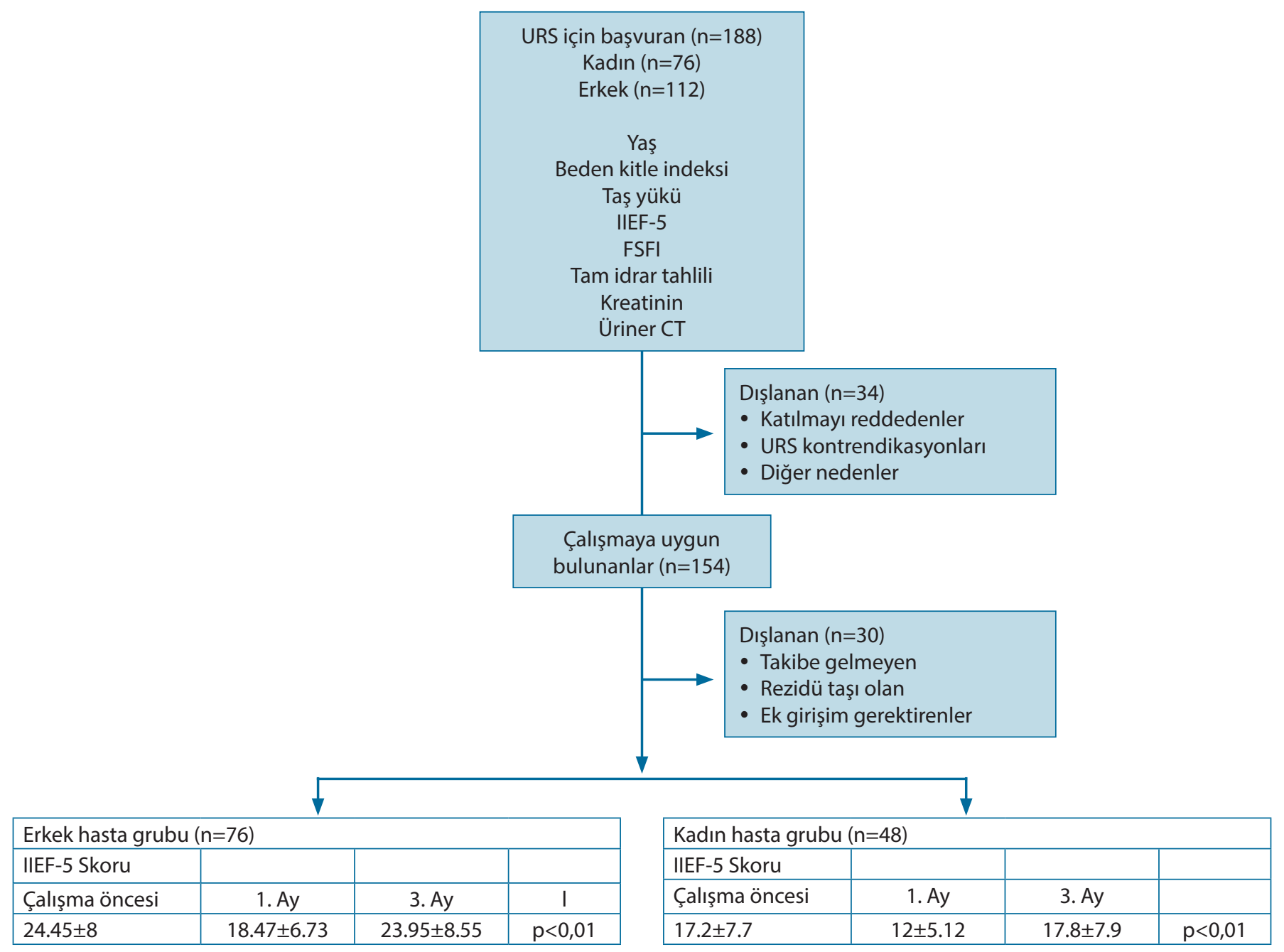

skorlama puanları, ortalama ve standart sapma olarak sunulmuştur. Başlangıç, birinci ay ve işlemden sonraki üçüncü ay anket puanları Student's t testi kullanılarak karşılaştırıldı. Skorlamaların ilk ölçümleri ile birinci ay ölçümleri arasındaki değişim oranı, paydaya ilk ölçüm ve paya iki ölçüm arasındaki fark yerleştirerek hesaplandı. Sonuç daha sonra yüzdeyi elde etmek için 100 ile çarpıldı. Formülün özeti $(\Delta$ takip/ilk ölçüm $) * 100$ 'dür. Her iki cinsiyet için JJ stentli ve stentsiz hastalardaki değişim yüzdesinin korelasyonları yapıldı. Karşılaştırmalı farklılıklar, $\mathrm{p}<0,05$ 'te istatistiksel olarak anlamlı kabul edildi.

\section{BULGULAR}

Erkek hastalarda ortalama yaş $38,2 \pm 9,9$, BMI $26,2 \pm 3,3$ ve taş yükü $64,1 \pm 31,2$ idi. Erkek hastaların IIEF-5 skorları ortalaması işlem öncesi $24,45 \pm 8$ iken birinci ayda $18,47 \pm 6,73$ 'e düşmüş üçüncü ayda ise $23,95 \pm 8,55^{\prime}$ 'e çımıştır. Erkek hastalarda işlem öncesi değerler ile birinci ay değerleri ve birinci ay değerleri ile üçüncü ay değerleri istatistiksel olarak anlamlı derecede farklı bulunmuştur $(\mathrm{p}<0,01)$ ancak işlem öncesi değerler ile üçüncü ay değerleri benzer bulunmuştur ( $\mathrm{p}>0,05)$ (Tablo 1). Erkek hastalardan 36'sına stent takılmamış 40'ına JJ stent tatbik edilmiştir. Stent takılmayan hastaların birinci ay IIEF-5 skorları değişim oranı $\% 23,33(24,25 \pm 3,3$ 'ten $18,59 \pm 2,2$ 'ye) iken stent takılan hastaların değişim oranı \%31,12 (25,74 $43,8^{\prime}$ ten $17,72 \pm 3,1$ 'e) olarak tespit edilmiş ve oranlar arasındaki fark istatistiksel olarak anlamlı derecede farklı bulunmuştur $(\mathrm{p}<0,05)$ (Tablo 2).

Kadın hastalarda ise ortalama yaş $41,28 \pm 9,1$, BMI $27 \pm 3,7$ ve taş yükü $67 \pm 27,5$ idi. Demografik veriler erkek ve kadın hastalar arasında benzer bulundu $(\mathrm{p}>0,05)$. Kadın hastaların ortalama FSFI değerleri ise işlem öncesi 17,2 $\pm 7,7$ iken birinci ayda $12 \pm 5,12$ ye düşmüş, üçüncü ayda ise $17,8 \pm 7,9$ 'a yükselmiştir. Kadın hastalarda işlem öncesi değerler ile birinci ay değerleri ve birinci ay değerleri ile üçüncü ay değerleri istatistiksel olarak anlamlı derecede farklı bulunmuştur $(\mathrm{p}<0,01)$ ancak işlem öncesi değerler ile üçüncü ay değerleri benzer bulunmuştur ( $\mathrm{p}>0,05)$ (Tablo 1). Kadın hastalardan 20'sine stent takılmamış 28 'ine JJ stent tatbik edilmiştir. Stent takılmayan hastaların birinci ay FSFI skorları değişim oranı $\% 21,25$ 
$(16,49 \pm 2,9$ 'dan $12,98 \pm 2,1$ 'e) iken stent tak1lan hasta-

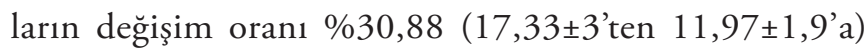
olarak tespit edilmiş ve oranlar arasındaki fark istatistiksel olarak anlamlı derecede farklı bulunmuştur $(\mathrm{p}<0,05)$ (Tablo 2).

Tablo 1. Hastaların demografik verileri ve üreterorenoskopi öncesi ve sonrası seksüel fonksiyonlar

\begin{tabular}{|c|c|c|c|c|}
\hline & \multicolumn{2}{|c|}{$\begin{array}{c}\text { Erkek } \\
\text { hastalar (n: 76) }\end{array}$} & $\begin{array}{c}\text { Kadın } \\
\text { hastalar (n: 48) }\end{array}$ & $p$ \\
\hline Yaş (yıl) & \multicolumn{2}{|c|}{$38,2 \pm 9,9$} & $41,28 \pm 9,1$ & 0,68 \\
\hline BMI $\left(\mathrm{kg} / \mathrm{m}^{2}\right)$ & \multicolumn{2}{|c|}{$26,2 \pm 3,3$} & $27 \pm 3,7$ & 0,44 \\
\hline \multirow[t]{2}{*}{ Taş yükü $\left(\mathrm{mm}^{2}\right)$} & \multicolumn{2}{|c|}{$64,1 \pm 31,2$} & $67 \pm 27,5$ & 0,47 \\
\hline & $\begin{array}{l}\text { URS } \\
\text { öncesi }\end{array}$ & $\begin{array}{l}\text { Birinci ay } \\
\text { takibi }\end{array}$ & $\begin{array}{l}\text { Üçüncü ay } \\
\text { takibi }\end{array}$ & $p$ \\
\hline $\begin{array}{l}\text { Erkek hastalar için } \\
\text { IIEF-5 skoru }\end{array}$ & $24,45 \pm 8$ & $18,47 \pm 6,73$ & $23,95 \pm 8,55$ & $<0,001$ \\
\hline $\begin{array}{l}\text { Kadın hastalar için } \\
\text { FSFI skoru }\end{array}$ & $17,2 \pm 7,7$ & $12 \pm 5,12$ & $17,8 \pm 7,9$ & $<0,001$ \\
\hline
\end{tabular}

BMI, beden kitle endeksi; URS, üreterorenoskopik üreter taşı tedavisi; IIEF, Uluslararası erektil işlev formu; FSFI, kadın cinsel işlev ölçeği.

Tablo 2. URS sonrası JJ takılmasının seksüel fonksiyonlar üzerine etkisi.

\begin{tabular}{lccc} 
Cinsiyet & JJ & JJ & \\
& takılan & takılmayan & $p$ \\
\hline $\begin{array}{l}\text { Erkek hastalarda IIEF-5 değişim } \\
\text { yüzdesi (\%) }\end{array}$ & 31,12 & 23,33 & $<0,001$ \\
$\begin{array}{l}\text { Kadın hastalarda FSFI değişim } \\
\text { yüzdesi (\%) }\end{array}$ & 30,88 & 21,25 & $<0,001$ \\
\hline
\end{tabular}

BMI, beden kitle endeksi; URS, üreterorenoskopik üreter taşı tedavisi; IIEF, Uluslararası erektil işlev formu; FSFI, kadın cinsel işlev ölçeği.

\section{TARTIȘMA}

Üreteroskopik prosedürlerin 1980 'li ylllardan beri hem üreter taşlarında hem de diğer üreter patolojilerinde kullanılması üroloji alanındaki en önemli gelişmelerden birisidir. ${ }^{[4]}$ URS geçen yıllar içerisinde hızla yaygınlaşarak tüm dünyada taş cerrahisinde primer tedavi modalitelerinden biri haline gelmiştir. Fleksibl üreteroskopların kullanıma girmesiyle bugün hem çocuklarda hem erişkinlerde distal üreter taşlarından intrarenal taşlara kadar geniş bir kullanım alanı bulmuştur. ${ }^{[26,27]}$ Yapılan çalışmalarda üreter taşlarında URS'nin taşsızlık oranları açısından en üstün yöntem olduğu bildirilmiştir. ${ }^{[28,29]}$ URS her ne kadar minimal invaziv ve yüksek başarı oranlarına sahip bir prosedür olsa da \%9-25 komplikasyon oranları bildirilmiştir. Bu komplikasyonların çoğu minör komplikasyonlardan oluşur ve ek müdahale gerektirmezken, \%1'den daha az görülen üreter avulsiyonu ve darlıklar ciddi komplikasyonları oluşturur. ${ }^{[30]}$
URS sonrası JJ stent uygulaması yaygın olarak kullanılmaktadır. JJ stentlerin yol açtı̆̆ı irritatif semptomlar, suprapubik ve flank ağrıların mekanizması tam olarak bilinmese de nöronlardan zengin mesane trigon mukozasının irritasyonu ve JJ stentin neden olduğu reflü bu semptomlarla ilişkilendirilmektedir. ${ }^{[20]}$ Üreteral stentlere bağlı bu semptomlar hastalar üzerinde anksiyete, depresyon ve yaşam kalitesinde düşüşe sebep olabilmektedir. Damiano ve ark., yaptıkları çalışmada üreteral stentlerin çaplarından bağımsız olarak anksiyete, depresyon ve yaşam kalitesi üzerinde düşüşe etkisi olduğunu göstermiştir. [31] Joshi ve arkadaşlarının yaptığı bir çalışmada üreteral stent takılan hastaların \%32'sinde cinsel fonksiyonların olumsuz etkilendiği, \%80'den fazla hastada ise üreteral stent nedenli ağrının günlük aktivitelerini olumsuz yönde etkilediği saptanmıştır. ${ }^{[32]}$ Sofer ve ark., erkek hastalar üzerinde yaptıkları çalışmalarında URS sonrası geçici bir seksüel disfonksiyon bildirmişler ve JJ stent takılan hastalarda seksüel disfonksiyonun daha belirgin olduğunu göstermişlerdir. ${ }^{[33]}$ Çok daha yeni bir meta-analizde JJ stent yerleştirilmesinin seksüel fonksiyonlar üzerine geçici bir etkisi olduğunu ancak kesin bir sonuca varmak için daha çok çalışmaya ihtiyaç olduğu bildirilmiştir. ${ }^{[34]}$ Bu meta-analizde ortaya konulduğu gibi JJ stentlerin bireylerin seksüel fonksiyonlarını etkileyip etkilemediğini konusunda literatüre katkı sağlamak için çalışmamızı geliştirdik ve sonuç olarak hem erkeklerde hem de kadınlarda URS'nin geçici seksüel disfonksiyona yol açtığı ve JJ stent tatbikinin seksüel fonksiyonlarda daha fazla bozulmaya yol açtığını ortaya koyduk. Bu durum URS ve sonrasında JJ stent uygulanan hastalarda ortaya çıkabilecek olan ağrı, alt üriner sistem semptomları yanında yine oluşabilecek uykusuzluk, anksiyete gibi duygudurumu etkileyebilecek faktörlere bağlı olabilir. Ancak bu seksüel disfonksiyonun kısa bir dönem sonrast (üçüncü ay) düzelmesi durumun geçici olduğunu göstermektedir. Çalışmamızın sınırlılıkları olarak hasta popülasyonunun küçüklüğü ve uzun dönem sonuçların bilinmemesi olarak sayılabilir. Bununla birlikte Beck depresyon indeksi veya Visuel analog skala gibi bir ağrı indeksinin doldurulmamış olması geçici seksüel disfonksiyonun nedeninin daha net ortaya konulmasının önüne geçmiştir.

Sonuç olarak URS uygulanacak hastalarda geçici de olsa seksüel disfonksiyon gelişebileceği hakkında hastalara bilgi verilmelidir. URS sonrası JJ stent takılan hastalarda seksüel fonksiyonlarda daha fazla kötüleşme görülebileceği akılda tutulmalıdır. Elde ettiğimiz sonuçların ileriki ve daha geniş hasta gruplarıyla yapılacak olan çalışmalarla desteklenmesi gerekmektedir. 
Etik Kurul Onayı

Çalışma, Gaziosmanpaşa Eğitim ve Araştırma Hastanesi Klinik Araştırmalar Etik Kurulu tarafından onaylandı. (onay tarihi ve sayısı: 23.09.2020/166).

\section{Hakem Değerlendirmesi}

Dış bağımsız.

Çıkar Çatışması

Yazarlar çıkar ilișkisi olmadığını beyan etmișlerdir.

\section{Finansal Destek}

Herhangi bir mali destek alınmamıştır.

\section{Ethics Committee Approva}

The study was approved by Gaziosmanpaşa Training and Research Hospital Clinical

Research Ethics Committee. (date and number of approval: 23.09.2020/166).

\section{Peer-review}

Externally peer-reviewed.

\section{Conflict of Interest}

No conflict of interest was declared by the authors.

Financial Disclosure

No financial support has been received.

\section{KAYNAKLAR}

1. Kittanamongkolchai W, Vaughan LE, Enders FT, Dhondup T, Mehta RA, Krambeck AE, et al. The changing incidence and presentation of urinary stones over 3 decades. Mayo Clin Proc 2018;93:291-9. [CrossRef]

2. Tseng TY, Preminger GM. Kidney stones. BMJ Clin Evid 2011;2011:2003. https://www.ncbi.nlm.nih.gov/pmc/articles/ PMC3275105/

3. Turk C, Neisius A, Petrik A, Seitz C, Skolarikos A, Somani B, et al. EAU guidelines on urolithiasis. EAoUG Office EAU guidelines (published as the 35th EAU Annual Meeting). Amsterdam, The Netherlands: European Association of Urology Guidelines Office; 2020.

4. Goodman TM. Ureteroscopy with rigid instruments in the management of distal ureteral disease. J Urol 1984;132:250-3. [CrossRef]

5. Ozturk MU, Şener NC, Goktug HG, Gucuk A, Nalbant I, İmamoglu MA. The comparison of laparoscopy, shock wave lithotripsy and retrograde intrarenal surgery for large proximal ureteral stones. Can Urol Assoc J 2013;7:E673-6. [CrossRef]

6. Castro EP, Osther PJ, Jinga V, Razvi H, Stravodimos KG, Parikh K, et al. Differences in ureteroscopic stone treatment and outcomes for distal, mid-, proximal, or multiple ureteral locations: the Clinical Research Office of the Endourological Society ureteroscopy global study. Eur Urol 2014;66:102-9. [CrossRef]

7. De La Rosette J, Denstedt J, Geavlete P, Keeley F, Matsuda T, Pearle $\mathrm{M}$, et al. The clinical research office of the endourological society ureteroscopy global study: indications, complications, and outcomes in 11, 885 patients. J Endourol 2014;28:131-9. [CrossRef]

8. Yafi FA, Jenkins L, Albersen M, Corona G, Isidori AM, Goldfarb S, et al. Erectile dysfunction. Nat Rev Dis Primers 2016;2:16003. [CrossRef]

9. Parish SJ, Goldstein AT, Goldstein SW, Goldstein I, Pfaus J, Clayton AH, et al. Toward a More evidence-based nosology and nomenclature for female sexual dysfunctions-Part II. J Sex Med 2016;13:1888-906. [CrossRef]

10. Dosch A, Rochat L, Ghisletta P, Favez N, Van der Linden M. Psychological Factors Involved in Sexual Desire, Sexual Activity, and Sexual Satisfaction: A Multi-factorial Perspective. Arch Sex Behav 2016;45:2029-45. [CrossRef]

11. Montejo AL, Montejo L, Baldwin DS. The impact of severe mental disorders and psychotropic medications on sexual health and its implications for clinical management. World Psychiatry 2018;17:3-11. [CrossRef]
12. Gandhi J, Dagur G, Warren K, Smith NL, Sheynkin YR, Zumbo A, Khan SA. The Role of Diabetes Mellitus in Sexual and Reproductive Health: An Overview of Pathogenesis, Evaluation, and Management. Curr Diabetes Rev 2017;13:573-81. [CrossRef]

13. Hendren SK, O’Connor BI, Liu M, Asano T, Cohen Z, Swallow $\mathrm{CJ}$, et al. Prevalence of male and female sexual dysfunction is high following surgery for rectal cancer. Ann Surg 2005;242:212-23. [CrossRef]

14. Rees PM, Fowler CJ, Maas CP. Sexual function in men and women with neurological disorders. Lancet 2007;369:512-25. [CrossRef]

15. Barlow DH. Causes of sexual dysfunction: the role of anxiety and cognitive interference. J Consult Clin Psychol 1986;54:140-8. [CrossRef]

16. Laurent SM, Simons AD. Sexual dysfunction in depression and anxiety: conceptualizing sexual dysfunction as part of an internalizing dimension. Clin Psychol Rev 2009;29:573-85. [CrossRef]

17. Incrocci L, Jensen PT. Pelvic radiotherapy and sexual function in men and women. J Sex Med 2013;10 Suppl 1:53-64. [CrossRef]

18. Akbal C, Türker P, Tavukçu HH, Simşek F, Türkeri L. Erectile function in prostate cancer-free patients who underwent prostate saturation biopsy. Eur Urol 2008;53:540-4. [CrossRef]

19. Kalkanli A, Gezmis CT. The Effect of Shock Wave Lithotripsy on Male and Female Sexual Dysfunction in Patients with Proximal Ureteral Stones. Urol Int 2021;105:963-8. [CrossRef]

20. Kuyumcuoglu U, Eryildirim B, Tuncer M, Faydaci G, Tarhan F, Ozgül A. Effectiveness of medical treatment in overcoming the ureteral double-J stent related symptoms. Can Urol Assoc J 2012;6:E234-7. [CrossRef]

21. Damiano R, Oliva A, Esposito C, De Sio M, Autorino R, D'Armiento M. Early and late complications of double pigtail ureteral stent. Urol Int 2002;69:136-40. [CrossRef]

22. Joshi HB, Newns N, Stainthorpe A, MacDonagh RP, Keeley FX Jr, Timoney AG. Ureteral stent symptom questionnaire: development and validation of a multidimensional quality of life measure. J Urol 2003;169:1060-4. [CrossRef]

23. Leibovici D, Cooper A, Lindner A, Ostrowsky R, Kleinmann J, Velikanov S, et al. Ureteral stents: morbidity and impact on quality of life. Isr Med Assoc J 2005;7:491-4. https://www.ima.org.il/ FilesUploadPublic/IMAJ/0/50/25308.pdf

24. Turunç T, Deveci S, Güvel S, Peşkircioğlu L. The assessment of Turkish validation with 5 question version of International Index of Erectile Function (IIEF-5). Turk J Urol 2007;33:45-9. https://turkishjournalofurology.com/content/files/sayilar/26/ buyuk/045-0491.pdf

25. Oksuz E, Malhan S. Reliability and validity of the Female Sexual Function Index in Turkish population. Sendrom 2005;17:5460. https://www.researchgate.net/publication/285879607_ Reliability_and_validity_of_the_Female_Sexual_Function_ Index_in_Turkish_population

26. Xiao J, Wang X, Li J, Wang M, Han T, Zhang C, et al. Treatment of upper urinary tract stones with flexible ureteroscopy in children. Can Urol Assoc J 2019;13: E78-82. [CrossRef]

27. Rob S, Jones P, Pietropaolo A, Griffin S, Somani BK. Ureteroscopy for Stone Disease in Paediatric Population is Safe and Effective in Medium-Volume and High-Volume Centres: Evidence from a Systematic Review. Curr Urol Rep 2017;18:92. [CrossRef]

28. Shah OD, Matlaga BR, Assimos DG. Selecting Treatment for Distal Ureteral Calculi: Shock Wave Lithotripsy versus Ureteroscopy. Rev Urol 2003;5:40-4. https://www.ncbi.nlm.nih.gov/pmc/articles/ PMC1472989/ 
29. Ghalayini IF, Al-Ghazo MA, Khader YS. Extracorporeal shockwave lithotripsy versus ureteroscopy for distal ureteric calculi: efficacy and patient satisfaction. Int Braz J Urol 2006;32:656-64; discussion 664-7. [CrossRef]

30. Perez Castro E, Osther PJ, Jinga V, Razvi H, Stravodimos KG, Parikh K, et al. Differences in ureteroscopic stone treatment and outcomes for distal, mid-, proximal, or multiple ureteral locations: the Clinical Research Office of the Endourological Society ureteroscopy global study. Eur Urol 2014;66:102-9. [CrossRef]

31. Damiano R, Autorino R, De Sio M, Cantiello F, Quarto G, Perdonà $S$, et al. Does the size of ureteral stent impact urinary symptoms and quality of life? A prospective randomized study. Eur Urol 2005;48:673-8. [CrossRef]
32. Joshi HB, Stainthorpe A, MacDonagh RP, Keeley FX Jr, Timoney AG, Barry MJ. Indwelling ureteral stents: evaluation of symptoms, quality of life and utility. J Urol 2003;169:1065-9;discussion 1069. [CrossRef]

33. Sofer M, Yehiely R, Greenstein A, Bar-Yosef Y, Matzkin H, Chen J. Endourological procedures and sexual dysfunction: a prospective multivariate analysis. BJU Int 2012;109:250-3. [CrossRef]

34. Lu J, Lu Y, Xun Y, Chen F, Wang S, Cao S. Impact of Endourological procedures with or without double-J stent on sexual function: a systematic review and meta-analysis. BMC Urol 2020;20:13. [CrossRef] 